\title{
Tumor microenvironment: becoming sick of Myc
}

\author{
Jonathan R. Whitfield • Laura Soucek
}

Received: 26 July 2011/Revised: 3 October 2011/Accepted: 5 October 2011/Published online: 28 October 2011

(C) The Author(s) 2011. This article is published with open access at Springerlink.com

\begin{abstract}
Several years ago, we described Myc as "the oncogene from hell", since evidence had just emerged that Myc, aside from being responsible for cell-cycle progression and tumor expansion, was also able to induce genomic instability in culture, wreaking havoc in tumor cells and accelerating tumor progression (Soucek and Evan, Cancer Cell 1:406-408, 2002; Vafa et al., Mol Cell 9:1031-1044, 2002). In this review, we discuss recent publications that expand Myc's evil armory to include coordination of the crosstalk between tumor and microenvironment. Indeed, endogenous Myc, acting as a client for upstream oncogenic lesions, instructs the tumor stroma, engages a complex inflammatory response and induces angiogenesis, thus allowing the tumor to thrive. This is highly topical in light of the fact that Hanahan and Weinberg have recently redefined the hallmarks of cancer and pointed out that genomic instability and inflammation are essential for both their acquisition and development (Hanahan and Weinberg, Cell 144:646-674, 2011). Myc, it seems, is behind it all.
\end{abstract}

Keywords Myc - Omomyc C Cancer .

Microenvironment · Tumorigenesis · Inflammation ·

Angiogenesis

\begin{abstract}
Abbreviations
VEGF Vascular endothelial growth factor

IL-1 $\beta \quad$ Interleukin-1 $\beta$
\end{abstract}

J. R. Whitfield · L. Soucek $(\bowtie)$

Vall d'Hebron Institute of Oncology (VHIO),

Psg. Vall d'Hebron 119, Edifici Mediterranea,

Laboratorio 20, 08035 Barcelona, Spain

e-mail: Lsoucek@vhio.net

J. R. Whitfield

e-mail: jwhitfield@vhio.net
Hif1a Hypoxia-inducible transcription factor 1 alpha

Bcl-xl B-cell lymphoma-extra large

RIP Rat insulin promoter 1

SV40 Simian virus 40

\section{Introduction}

Myc is a highly pleiotropic transcription factor known to control proliferation, metabolism, differentiation, and apoptosis [4-6]. Normally its expression is tightly regulated. In human cancer, however, Myc's deregulated expression is often observed and is considered a poor prognostic factor [7-10]. Myc is evolutionarily conserved as an integrator of extracellular and intracellular signals leading to cell growth and division, tissue regeneration, and remodeling [11]. Indeed, during development, myc family gene expression is highest during embryonic stages and is downregulated in mature organs, due to cell growth arrest and differentiation [12].

Genetic knockout of the $c-m y c$ gene leads to embryonic lethality and, as elegantly shown by Baudino et al. [13], this is partially due to defects in vasculogenesis caused by the lack of proper Vascular Endothelial Growth Factor (VEGF) signaling [13]. In the same study, a role in murine embryonic stem cells and derived teratomas was attributed to Myc in the regulation of other proteins strictly involved in angiogenesis, such as thrombospondin-1 and angiopoietin-1 and -2, providing the first evidence to show Myc as a master regulator of vascular remodeling [13]. Myc's role in embryonic development has recently been defined more precisely by the use of cell lineage specific deletion [14]: embryos lacking c-myc in both endothelial and hematopoietic compartments phenocopied those lacking 
$c$-myc in the entire embryo, as a consequence of defective hematopoiesis and vasculogenesis. Surprisingly though, $c$-myc deletion in endothelial cells alone did not prevent endothelial cell proliferation and vasculogenesis. Thus, it is c-myc-mediated hematopoiesis that is critical for blood vessel formation during mammalian development [14].

Further evidence for the regulation of VEGF and angiogenesis by Myc was provided by Mezquita and colleagues [15], who demonstrated that a human B-cell line increases VEGF production tenfold upon Myc overexpression, due to increased initiation of VEGF mRNA translation. Ngo et al. [16] confirmed in vivo Myc's down-modulation of thrombospondin-1 by making use of Myc-transformed Rat1A fibroblasts that form vascular tumors in immunocompromised mice. Finally, Dews and colleagues [17] suggested that thrombospondin-1 is downregulated by Myc through induction of the miR-17-92 microRNA cluster, using p53-null mouse colonocytes transformed in vitro by low-grade overexpression of activated K-Ras and Myc, and engrafted into the cecal wall of syngeneic mice. Collectively, these results show that Myc efficiently modulates in vivo various potent factors implicated in neoangiogenesis.

Another giant leap forward in understanding Myc's role as instructor of the tumor microenvironment was made using mouse models of Myc-induced tumorigenesis. In particular, the use of reversibly switchable models that allowed synchronous activation or deactivation of Myc also permitted deconvolution of the cause-and-effect processes consequent to activation of the oncogene in vivo. One of these models is the pInsMycER ${ }^{\text {Tam }} ; R I P-B c l X L$ model of pancreatic $\beta$-cell tumorigenesis, in which expression of a MycER fusion protein is spatially controlled by the tissue-specific insulin promoter ( $p$ Ins) and temporally regulated by the administration of 4-hydroxytamoxifen [18]. Apoptosis is prevented by co-expression of B-cell lymphoma-extra large $(B c l-x l)$ driven from the Rat insulin promoter $1(R I P)$. In this model, Shchors et al. demonstrated that acute Myc activation in $\beta$-cells triggers the release of factors that induce proliferation of adjacent endothelial cells, and their subsequent formation of leaky and complex vessels [19]. This process is mediated by the release of interleukin- $1 \beta$ (IL- $1 \beta$ ) by $\beta$-cells, which leads to mobilization of VEGF-A bound to the extracellular matrix, presumably through the action of extracellular proteases $[19,20]$.

In the same mouse model, we showed that Myc alone causes a complex inflammatory response, leading to the recruitment of various inflammatory cells. Among those, mast cells are absolutely required for tumor expansion and sustained proliferation of endothelial cells within the tumor [21]. We also showed that inhibitors of mast cell function rapidly triggered hypoxia and cell death in tumors and vessels, which suggests that there are Myc effectors in the tumor stroma, offering new therapeutic opportunities [21]. These data underscore the essential role of the activation of inflammatory pathways for Myc's oncogenic activity and, together with the previous work by Shchors et al. [19], demonstrate that Myc can directly instruct tissue remodeling, angiogenesis and inflammation.

Other studies have suggested the intriguing possibility of bi-directional crosstalk between Myc-driven tumors and their microenvironment. Giuriato et al. [22] and Rakhra et al. [23] showed that, in a conditional mouse model for Myc-induced tumorigenesis in hematopoietic cells, complete tumor regression, cellular senescence and shutdown of angiogenesis upon Myc inactivation can be achieved only through persistent expression of thrombospondin-1 [22] and in the presence of $\left.\mathrm{CD} 4{ }^{+}\right) \mathrm{T}$ cells [23].

Furthermore, hypoxia and other environmental stresses can affect c-Myc expression itself. For instance, low oxygen supply leads to stabilization of hypoxia-inducible transcription factor 1 alpha (Hif1a), whose transcriptional activity both antagonizes and cooperates with Myc [24], while low oxygen and glucose deficiency can destabilize Myc [25].

Yet, for a long time, Myc's interaction with the microenvironment was thought to be a prerogative of overexpressed and not physiological levels of Myc. More recently, we made use of a dominant-negative form of Myc, termed Omomyc, to assess the requirement for Myc activity in tumorigenesis. More specifically, we decided to study $\beta$-cell insulinomas arising in Rip-Tag2 mice that express Simian virus 40 (SV40) T/t antigens, the 'workhorse' for tumor microenvironment and angiogenic switch studies [26, 27]. We crossed these mice with the TREOmomyc;CMVrtTA strain, thus enabling controlled expression of Omomyc in most mouse organs [28]. This dominant-negative mutant interferes with Myc's transactivation activity [29], and we sought to establish the extent to which tumor angiogenesis is dependent upon endogenous Myc. Strikingly, given the enormous transforming potential of $\mathrm{T} / \mathrm{t}$ antigens, tumor expansion was completely prevented by Myc inhibition, and furthermore, tumors collapsed after induced expression of Omomyc [30]. Notably, at no stage of tumor evolution was Myc overexpressed, suggesting it functions simply_but critically-as a client for upstream SV40 oncoproteins. Similarly, in most human cancers, Myc does not appear to be mutated itself, but more typically is induced by altered signal transduction [31]. Our results show that, even in cases where it is not upregulated, Myc still has a crucial part to play in tumorigenesis. Indeed, inhibition of endogenous - neither mutated nor overexpressed-Myc has a huge impact on the tumor stroma: it impairs VEGF signaling, causes disappearance of infiltrating inflammatory cells and leads to vasculature 
collapse, all events that precede actual tumor regression [30]. Importantly, this holds true even when Myc is inhibited exclusively in tumor cells and not the microenvironment [30], showing that it is Myc in the tumor cells that directs changes in the tumor stroma.

This is particularly intriguing when considered together with recent data from Pello et al. [32], which define a clear role for Myc in controlling the activation of tumor-associated macrophages. This study indicates that Myc inhibition in the microenvironment also has therapeutic promise, being able to prevent alternative polarization of macrophages and their pro-tumorigenic behavior [32].

Various considerations follow:

First, since no emergence of resistance to Myc inhibition was observed in any tumor lesion, these results revealed a unique, non-adaptive link between tumor and microenvironment, which provides tremendous therapeutic opportunities.

Second, it remains to be established whether Myc's instruction of the microenvironment is conserved in different tumors and tissues, and downstream of different oncogenic lesions, or whether the degeneracy observed in signals upstream of Myc is also maintained in downstream pathways.

Third, Omomyc exerts an "edgetic" perturbation [33] of the Myc transcriptome [29, 34] - that is to say, Omomyc does not ablate all Myc activities but specifically Mycdependent gene transactivation, rather than transrespression [29, 34]. This contrasts with approaches designed to totally ablate the gene product function, such as gene knockout or RNA interference techniques. Hence, the microenvironmental effects elicited by Omomyc are critically dependent on the inhibition of Myc's transactivated target genes.

Finally, it is well known that metastasis is a multistage process that requires cancer cells to escape from the primary tumor, survive in the circulation, seed at distant sites, and grow. Each of these processes is influenced by non-malignant cells of the tumor microenvironment [35]. It remains to be established whether Myc is again a critical node during such stages of tumorigenesis and whether inhibiting Myc has therapeutic activity against metastasis.

In summary, instruction of the microenvironment, previously considered a feature of aberrant, mutated or overexpressed Myc, is more likely just one of Myc's many and diverse physiological cellular activities. This should come as no surprise if we consider Myc as a nodal, central, and non-redundant integrator of intracellular and extracellular programs normally involved in organogenesis and tissue regeneration. It is this same physiological activity that is hijacked during tumorigenesis, turning Myc from healing and regenerating saint, to sinner.
Open Access This article is distributed under the terms of the Creative Commons Attribution Noncommercial License which permits any noncommercial use, distribution, and reproduction in any medium, provided the original author(s) and source are credited.

\section{References}

1. Soucek L, Evan G (2002) Myc-Is this the oncogene from Hell? Cancer Cell 1:406-408

2. Vafa O, Wade M, Kern S et al (2002) c-Myc can induce DNA damage, increase reactive oxygen species, and mitigate p53 function: a mechanism for oncogene-induced genetic instability. Mol Cell 9:1031-1044

3. Hanahan D, Weinberg RA (2011) Hallmarks of cancer: the next generation. Cell 144:646-674

4. Grandori C, Cowley SM, James LP, Eisenman RN (2000) The $\mathrm{Myc} / \mathrm{Max} / \mathrm{Mad}$ network and the transcriptional control of cell behavior. Annu Rev Cell Dev Biol 16:653-699

5. Nasi S, Ciarapica R, Jucker R, Rosati J, Soucek L (2001) Making decisions through Myc. FEBS Lett 490:153-162

6. Albihn A, Johnsen JI, Henriksson MA (2010) MYC in oncogenesis and as a target for cancer therapies. Adv Cancer Res 107:163-224

7. Lonn U, Lonn S, Nilsson B, Stenkvist B (1995) Prognostic value of erb-B2 and myc amplification in breast cancer imprints. Cancer 75:2681-2687

8. Roncalli M, Viale G, Grimelius L et al (1994) Prognostic value of $\mathrm{N}$-myc immunoreactivity in medullary thyroid carcinoma. Cancer 74:134-141

9. Bourhis J, Le MG, Barrois M et al (1990) Prognostic value of c-myc proto-oncogene overexpression in early invasive carcinoma of the cervix. J Clin Oncol 8:1789-1796

10. Herms J, Neidt I, Luscher B et al (2000) C-MYC expression in medulloblastoma and its prognostic value. Int $\mathrm{J}$ Cancer 89:395-402

11. Gallant P (2006) Myc/Max/Mad in invertebrates: the evolution of the Max network. Curr Top Microbiol Immunol 302:235-253

12. Zimmerman KA, Yancopoulos GD, Collum RG et al (1986) Differential expression of myc family genes during murine development. Nature 319:780-783

13. Baudino TA, McKay C, Pendeville-Samain H et al (2002) c-Myc is essential for vasculogenesis and angiogenesis during development and tumor progression. Genes Dev 16:2530-2543

14. $\mathrm{He} \mathrm{C}, \mathrm{Hu} \mathrm{H}$, Braren $\mathrm{R}$ et al (2008) c-myc in the hematopoietic lineage is crucial for its angiogenic function in the mouse embryo. Development 135:2467-2477

15. Mezquita P, Parghi SS, Brandvold KA, Ruddell A (2005) Myc regulates VEGF production in $\mathrm{B}$ cells by stimulating initiation of VEGF mRNA translation. Oncogene 24:889-901

16. Ngo CV, Gee M, Akhtar N et al (2000) An in vivo function for the transforming Myc protein: elicitation of the angiogenic phenotype. Cell Growth Differ 11:201-210

17. Dews M, Homayouni A, Yu D et al (2006) Augmentation of tumor angiogenesis by a Myc-activated microRNA cluster. Nat Genet 38:1060-1065

18. Pelengaris S, Khan M, Evan GI (2002) Suppression of Mycinduced apoptosis in beta cells exposes multiple oncogenic properties of Myc and triggers carcinogenic progression. Cell 109:321-334

19. Shchors K, Shchors E, Rostker F, Lawlor ER, Brown-Swigart L, Evan GI (2006) The Myc-dependent angiogenic switch in tumors is mediated by interleukin 1beta. Genes Dev 20:2527-2538

20. Shchors K, Evan G (2007) Tumor angiogenesis: cause or consequence of cancer? Cancer Res 67:7059-7061 
21. Soucek L, Lawlor ER, Soto D, Shchors K, Swigart LB, Evan GI (2007) Mast cells are required for angiogenesis and macroscopic expansion of Myc-induced pancreatic islet tumors. Nat Med 13:1211-1218

22. Giuriato S, Ryeom S, Fan AC et al (2006) Sustained regression of tumors upon MYC inactivation requires $\mathrm{p} 53$ or thrombospondin1 to reverse the angiogenic switch. Proc Natl Acad Sci USA 103:16266-16271

23. Rakhra K, Bachireddy P, Zabuawala $\mathrm{T}$ et al $(2010) \mathrm{CD} 4\left(^{+}\right) \mathrm{T}$ cells contribute to the remodeling of the microenvironment required for sustained tumor regression upon oncogene inactivation. Cancer Cell 18:485-498

24. Gordan JD, Thompson CB, Simon MC (2007) HIF and c-Myc: sibling rivals for control of cancer cell metabolism and proliferation. Cancer Cell 12:108-113

25. Okuyama H, Endo H, Akashika T, Kato K, Inoue M (2010) Downregulation of c-MYC protein levels contributes to cancer cell survival under dual deficiency of oxygen and glucose. Cancer Res 70:10213-10223

26. Christofori G, Hanahan D (1994) Molecular dissection of multistage tumorigenesis in transgenic mice. Semin Cancer Biol $5: 3-12$

27. Bergers G, Hanahan D (2008) Modes of resistance to antiangiogenic therapy. Nat Rev Cancer 8:592-603
28. Soucek L, Whitfield J, Martins CP et al (2008) Modelling Myc inhibition as a cancer therapy. Nature 455:679-683

29. Soucek L, Jucker R, Panacchia L, Ricordy R, Tato F, Nasi S (2002) Omomyc, a potential Myc dominant negative, enhances Myc-induced apoptosis. Cancer Res 62:3507-3510

30. Sodir NM, Swigart LB, Karnezis AN, Hanahan D, Evan GI, Soucek L (2011) Endogenous Myc maintains the tumor microenvironment. Genes Dev 25:907-916

31. Nilsson JA, Cleveland JL (2003) Myc pathways provoking cell suicide and cancer. Oncogene 22:9007-9021

32. Pello OM, De Pizzol M, Mirolo M, Soucek L, Zammataro L, Amabile A, Doni A, Nebuloni M, Swigart LB, Evan GI, Mantovani A, Locati M. (in press) Role of c-Myc in alternative activation of human macrophages and tumor-associated macrophage biology. Blood

33. Zhong Q, Simonis N, Li QR et al (2009) Edgetic perturbation models of human inherited disorders. Mol Syst Biol 5:321

34. Savino M, Annibali D, Carucci N, Soucek L, Evan GI, Nasi S (2011) The action mechanism of the Myc inhibitor termed Omomyc may give clues on how to target Myc for cancer therapy. PLoS One 6(7):e22284

35. Joyce JA, Pollard JW (2009) Microenvironmental regulation of metastasis. Nat Rev Cancer 9:239-252 